\title{
Enteric Fever in Children at Dhulikhel Hospital
}

\author{
Singh DS ${ }^{1}$, Shrestha $\mathbf{S}^{2}$, Shrestha $\mathbf{N}^{3}$, Manandhar $\mathbf{S}^{4}$
}

${ }^{1}$ Dr. Srijana Dongol Singh, MBBS, MD, Assistant Professor, ${ }^{2}$ Dr. Shreema Shrestha, MBBS, MD, Assistant Professor, ${ }^{3}$ Dr. Narayan Shrestha, MBBS, MD, Lecturer, ${ }^{4}$ Dr. Sanjay Manandhar, MBBS, Medical Officer. All from the Department of Paediatrics, Dhulikhel Hospital, Kathmandu University Teaching Hospital, Dhulikhel, Nepal.

Address for correspondence: Dr. Srijana Dongol Singh, E-mail: dongolsrijana@gmail.com

\begin{abstract}
Introduction: Typhoid fever is one of the most common public health problems in Nepal. It occurs in all parts of the world where water supplies and sanitation are sub-standard. In Dhulikhel hospital, this is one of the top acute febrile illnesses in inpatient department. The objectives of this study were to evaluate the clinical and laboratory parameters including culture and sensitivity, the response to therapy, and complications of enteric fever among child cases at Dhulikhel Hospital. Materials and Methods: This retrospective study was conducted at Dhulikhel Hospital, Kathmandu University Teaching Hospital from January 2009 to June 2011. Statistical analysis was done with SPSS. Results: There were total of 138 cases of enteric fever admitted. There were 73 (53\%) male and 65 (47\%) female. Eighty-one percent were above five years of age. The most common clinical presentation was fever (100\%) followed by headache and G I symptoms. Hepatomegaly was the most common sign seen among the cases and was seen in 110 cases (79.71\%). Most of the patients had normal WBC count 100 (72.46\%) Widal test was positive in $70(50.72 \%)$ cases and blood culture was positive in 52(37.68\%) cases. Nalidixic acid was found to be resistant in 26 (50\%) cases. Complications were seen in only 7 (5\%) enteric fever cases. Conclusion: Typhoid fever is predominant in school going children in Nepal with slight male predominance. Fever lasting over 3 days followed by headache and Gl symptoms are the major presenting symptoms. In making the diagnosis, the isolation of bacteria from blood is the "gold standard". Nalidixic acid resistant Salmonella typhi is on the increasing trend. Pneumonia was found to be the most common complication among all other complications seen in enteric cases. In Dhulikhel Hospital this is one of the top acute febrile illnesses in inpatient department.
\end{abstract}

Key words: Antibiogram, Hepatomegaly, S. typhi, Splenomegaly, Typhoid fever

\section{Introduction}

Thes

yphoid or enteric fever is an ancient disease, which

has afflicted mankind since human populations grew large enough to contaminate their water and food supplies. It is a waterborne and food borne disease caused by Salmonella entirica serovar Typhi (S.typhi) and Salmonella entirica serovar Parathyphi (S.paratyphi).

The WHO conservatively estimates the annual global incidence of typhoid fever at 21 million cases, of which $1-4 \%$ end fatally. An estimated $90 \%$ of these deaths occur in Asia'. A recent epidemiologic study showed that south-east and south central Asia are the regions most endemic in the world with rates greater than 100/100,000 cases per year. The rest of Asia, Africa, Latin America, the Caribbean, and Oceania (expect Australia and New Zealand) are the next highest with incidence rates between 10-100/100,000 cases/year. Europe, North America, and the rest of the developed world have low rates of disease less than 10/100,000 cases/year ${ }^{2}$.

Enteric fever (locally known as Bisham Jworo or Myade Jworo) is endemic in Nepal and constitutes a mojor cause of morbidity and mortality ${ }^{3,4}$. The disease is predominantly a disease of school age children and young adults and is reported to be milder in infants and young children ${ }^{3,5}$. According to the WHO case definition, a probable case of typhoid fever is a patient with fever $38^{\circ} \mathrm{C}$ and above that lasted for at least 3 days with a positive serodiagnosis or antigen detection test but without S.typhi isolation. Typhoid fever confirmed case definition, laboratory confirmed positive culture must be present ${ }^{6}$. 
The primary sources of infection are feces and urine of cases or carriers; secondary sources include contaminated water, food, fingers, and flies. The disease may be acute non-complicated (characterized by prolonged fever, disturbance in bowel functions, headache, malaise, and anorexia) and complicated (intestinal bleeding, malena, intestinal perforation, and peritonitis) ${ }^{6}$.

The importance of enteric fever has increased once again due to the emergence of drug resistant strains. The spread of multidrug resistant S.typhi has reduced the number of effective treatment options, increased treatment costs, and resulted in higher rates of serious complication and deaths ${ }^{1}$. This emergence of multidrug resistant typhoid in the 1990s lead to widespread use of fluoroquinolones as the treatment of choice of suspected typhoid, especially in South Asia and Southeast Asia where the disease was endemic ${ }^{7}$. In recent years, however, the emergence of resistance to quinolones has placed tremendous pressure on public health system in developing countries as treatment options are limited ${ }^{8}$.

\section{Materials and Methods}

This retrospective study was conducted at Dhulikhel Hospital, Kathmandu University Teaching Hospital from January 2009 to June 2011. The study was approved by the Kathmandu University School of Health Science Institutional review board.

A total of 138 cases of suspected typhoid fever admitted in the paediatric ward were studied. The diagnostic criteria of typhoid fever were based on clinical and laboratory findings. The clinical findings were high grade fever over three days with headache, myalgia, cough, Gl symptoms, hepatomegaly, and splenomegaly. The laboratory findings included in this study were total WBC counts, blood culture positive for salmonella and Widal test with a cut off titer of 1:160. In all clinically suspected cases of Enteric fever, total and differential counts and blood cultures were sent. If child presented in the second week with features of Enteric fever, Widal test was sent. For culture and sensitivity tests, at least $5 \mathrm{ml}$ blood was used to collect and followed up to $7^{\text {th }}$ day of collection. Febrile illnesses of other causes were excluded with necessary investigation. If Widal test and blood cultures were both negative but patients with strong clinical suspicions and low counts were still considered as Enteric fever cases. Demographic data, clinical parameters, laboratory findings, antimicrobial use and their response, and complications were analyzed. The statistical analysis was done with Statistics software version 17.0.

\section{Results}

There were a total of 138 cases over the two and a half year period. There were 73 (53\%) males and 65 (47\%) females included in this study.

Their mean age was 8.5 years. The enteric cases were seen most frequently in children between 6-10 years followed by 11-15 years, and least frequently in children below 5 years of age. Among the study population, 81\% $(n=112)$ were school going children.

Table1: Showing the clinical feature and lab reports

\begin{tabular}{|l|c|c|}
\hline \multicolumn{1}{|c|}{ Parameter } & $\begin{array}{c}\text { Total no } \\
\text { (n=103) }\end{array}$ & $\begin{array}{c}\text { Percentage } \\
\text { (\%) }\end{array}$ \\
\hline Symptoms: & 138 & 100 \\
Fever & 100 & 72.4 \\
Headache & 66 & 48 \\
Anorexia & 54 & 39 \\
Cough & 46 & 33.3 \\
Abdominal pain & 38 & 27.5 \\
Nausea & 28 & 20.2 \\
Myalgia & 19 & 13.7 \\
Loose motion & 110 & 79.7 \\
\hline Signs: & 20 & 14.4 \\
Hepatomegaly & & \\
Splenomegaly & 16 & 12 \\
\hline Lab parameters: & 100 & 72.4 \\
Total count & 22 & 15.9 \\
$\quad$ Leukopenia & 70 & 50.7 \\
Normal count & 52 & 37.6 \\
Leukocytosis & & \\
\hline Widal test positive & & \\
\hline Blood culture positive & & \\
\hline
\end{tabular}

The clinical features and lab finding of enteric fever are shown in Table1.The most common symptom was fever, which was the presenting symptom of all cases $100 \%(n=138)$. Other symptoms were headache $72.4 \%(n=100)$, anorexia $48 \%(n=66)$, cough $39 \%(n=54)$, abdominal pain $33.3 \%(n=46)$, nausea $27 \%(n=38)$, myalgia 20\% $(n=28)$, and loose motion $13.7 \%(n=19)$. Among the signs, hepatomegaly $79.7 \%(n=110)$ was more common than splenomegaly $14.4 \%(n=20)$. In the paediatric age group, the liver may be palpable about 2 centimeters from the right costal margin as normal variant. Hepatomegaly in these cases may be a normal variant or as the result of enteric fever. Hepatomegaly was commonly seen between the ages of 5 to 10 years. In all cases total and differential count, widal and blood culture was done according to duration of illness. Most of the patients had normal blood counts $72 \%(n=100)$, while $16 \%(n=12)$ had leukocytosis and only $12 \%(n=16)$ cases had leucopenia. 
Widal test was positive in $50.7 \%(n=70)$ of cases while only $18 \%(n=19)$ had significant rise (i.e. four fold raise in titer). Among 138 cases, only 52 (38.6\%) had a positive blood culture when blood culture was follow up to seven days.

Table 2: Showing the antibiogram of culture positive cases $(n=52)$

\begin{tabular}{|l|c|c|c|}
\hline \multicolumn{1}{|c|}{ Drug } & Sensitive & $\begin{array}{c}\text { Partially } \\
\text { sensitive }\end{array}$ & Resistant \\
\hline Chloramphenicol & 42 & 0 & 10 \\
\hline Gentamycin & 49 & 0 & 0 \\
\hline Ciprofloxacin & 48 & 0 & 4 \\
\hline Cotrimoxazole & 43 & 6 & 2 \\
\hline Ampicillin & 11 & 7 & 34 \\
\hline Ceftriaxone & 50 & 1 & 0 \\
\hline Azithromycin & 50 & 2 & 0 \\
\hline Cefixime & 13 & 0 & 0 \\
\hline Nalidixic acid & 8 & 19 & 26 \\
\hline Amoxycillin & 3 & 0 & 0 \\
\hline Cefuroxime & 1 & 0 & 0 \\
\hline
\end{tabular}

Antimicrobial resistance was observed with ampicillin $65 \% \quad(n=34)$, nalidixic acid $50 \% \quad(n=26)$, chloramphenicol 19\% $(n=10)$, ciprofloxacin $8 \%(n=4)$ and cotrimoxazole $4 \%(n=2)$.

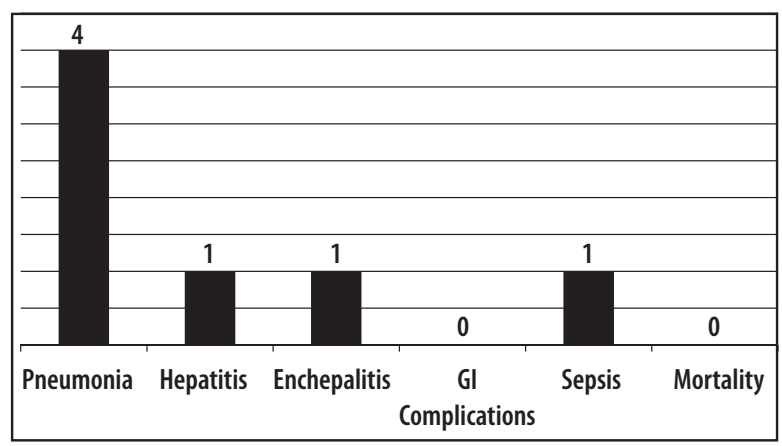

Fig 1: Showing various complications due to typhoid in among 138 cases.

Among the 138 cases, all were completely cured, but only 7 had complications as shown in Figure 1. The complications included 4 cases of pneumonia, 1 with enteric hepatitis, 1 with enteric encephalitis, and 1 with sepsis. There was no mortality among 138 observed cases.

During the hospital stay, the most common antimicrobial used was intravenous Ceftriaxone. Few cases were treated with other intravenous or oral medications. (azithromycin, cefixime, and ciprofloxacin)

\section{Discussion}

Enteric fever is a major health problem in developing countries attributed to poor sanitary and hygienic condition including a lack of potable water. Investigation from the US Centers for Disease Control and Prevention estimate that there are 21.6 million typhoid cases annually, with the annual incidence varying from 100 to 1000 cases per 100,000 population ${ }^{2}$. The global mortality estimates from typhoid have also been revised downwards from 600,000 to 200,000 , largely on the basis of regional extrapolations ${ }^{2}$.

In the present study, 138 cases of acute febrile illness, with other strong clinical features were analyzed along with supportive lab reports. In this study, 19\% $(n=26)$ of patients with typhoid fever were under 5 years, and the most affected group $81 \%(n=112)$ were school children, which was close to figures of other studies ${ }^{9,10}$.

Enteric fever was more common in the male population than in the female population. Similar results were seen in the study done by Malla T et al ${ }^{11}$ in Manipal Hospital. Male predominance was also seen in another study done by Shakya KN et al $^{5}$ in Kathmandu Medical College in 2008.

Children in the study group commonly presented with fever $100 \%(n=138)$, headache $72.4 \%(n=100)$ and gastrointestinal symptoms $48 \%(n=66)$. In this study diarrhea was more common than constipation, which is in accordance with the results from other studies ${ }^{11}$. The recent study done in India by Dhree G et al in 2012 also shows that fever with $\mathrm{Gl}$ symptoms, including diarrhea, are the most common clinical presentation in children with enteric fever ${ }^{12}$. Among the study population, hepatomegaly was seen in $79.7 \% \quad(n=110)$ and splenomegaly was seen in only $14.4 \%(n=20)$ of cases, which was similar to another study in India ${ }^{12,13}$.

Most of the cases in this study $72.5 \%(n=100)$ had normal total leukocyte counts, only $11.5 \%(n=16)$ cases had leucopenia, and $16 \%(n=22)$ cases had leukocytosis, which was similar to another study done in India ${ }^{14}$ where leucopenia was seen in $8 \%$ of the cases and leukocytosis in $12 \%$ of the cases. Another study done by Sharma $\mathrm{N}$ et $\mathrm{al}^{3}$ and his colleague (at Dhulikhel hospital in 2003) also showed that about $62.5 \%$ of cases had normal leukocyte count.

Widal test was positive in $50.7 \%(n=70)$ of cases similar to earlier study by Sharma $\mathrm{N}$ et $\mathrm{al}^{3}$. Unlike this study, positive widal test was higher in another study ${ }^{11}$ done in Manipal Teaching Hospital, Pokhara where Widal test was positive in $83 \%$ of the cases. The growth positive rate for Salmonella Typhi in this study was the 52 (37.6\%) which is high in comparison to the $5.4 \%$ reported in a study done by $\mathrm{P}$ Pokhrel et $\mathrm{al}^{15}$ from Nepal Medical College and Khanal et $\mathrm{al}^{4}$ from a teaching hospital in eastern Nepal reported $5.1 \%$. In another study done by 
Amatya et $\mathrm{al}^{16}$ from Kathmandu, a positive Salmonella culture rate of $23.1 \%$ was reported.

The last two decades have seen a change in the pattern of enteric fever with the emergence of multidrugresistant strains (MDRS). The emergence of antimicrobial resistance during the last two and half years was also observed in this study. $65 \%$ were resistant to ampicillin, $50 \%$ to nalidixic acid, $19 \%$ to chloramphenicol, $8 \%$ to ciprofloxacin and $4 \%$ to cotrimoxazole. There was no resistance to ceftriaxone, cefuroxime, ofloxacin and gentamycin. Multidrug resistance (resistance to ampicillin, trimethoprim/sulphamethoxazole and chloramphenicol) was also not seen in this study which is in contrast to other studies done in different countries like $63 \%$ in Ghana, $7 \%$ in India, 22\% in Vietnam and $65 \%$ in Pakistan ${ }^{18,19}$. In the present study S. typhi was most resistant to ampicillin. Another study done by Amatya NM in Nepal in 2007 showed that all isolates of Salmonella typhi was susceptible to ceftriaxone, which is consistent with the present study ${ }^{20}$. In the same study, it was also concluded that chloramphenicol was the foremost drug of choice among the tested antibiotics with its sensitivity rate of $98.4 \%$, which is slightly higher than the present study ${ }^{20}$. In contrast to this study, another study from Nigeria showed that among sero Typhi strains isolated from hospitalized patients in Lagos during 1997-2004, resistance rates reached $87 \%$ from ampicillin. Nalidixic acid resistance was found in $50 \%$ of patients among the culture positive cases in this present study. In another study done by Ochial LR et $\mathrm{al}^{21}$ in five different countries in Asia, showed a significant increase in nalidixic acid resistance in 59\% of isolates from the sites in Pakistan, 57\% from those in India, and 44\% from those in Vietnam $(p<0.0001)$ from overall heterogeneity of these proportions among the five sites. S. typhi was less resistant to cotrimoxazole, ciprofloxacin, and chloramphenicol followed by others. The finding was closer to previous findings from $\mathrm{Nepal}^{3,23 .}$ There was no resistance to ceftriaxone and ofloxacin and this finding was similar to the previous studies done in Nepal ${ }^{3,5,11}$.

There was no mortality in this series. Complications of typhoid fever were seen in $5 \%$ of children with Enteric fever. A similar result was seen in the study done in Chennai-South India where complications were seen in $4 \%$ of children ${ }^{14}$. The complications seen in this series were less in comparison to other studies, where complications occurred in $10-15 \%$ cases $^{14,22}$.

Nepal is considered as the Enteric fever endemic county and still known as an enteric fever capital of the world ${ }^{23}$ with the significant burden of enteric fever within the local population and in travelers visiting the area. The same study also showed no significant signs of decreasing pattern of enteric fever in the country.
Typhidot $M$ and Diazo test are good screening tests for early diagnosis of typhoid fever. One recent study done by Beig FK et $\mathrm{al}^{24}$ in 2010 showed Typhidot $M$ is superior to Diazo but the latter is more suitable to resource poor settings being economic and easy to perform.

\section{Conclusion}

Typhoid fever continues to be a major health problem resulting in significant number of children requiring hospitalization. In Dhulikhel Hospital this is one of the top causes of acute febrile illness in the inpatient department.

Typhoid fever is predominant in school going children with slight male predominance. Fever over 3 days followed by headache and Gl symptom are major presenting symptoms. In making the diagnosis, the isolation of bacteria from blood is the "gold standard" but widespread uncontrolled use of antimicrobial leads to negative culture results. In this situation, Widal test and white blood cell count play a supportive role in diagnosis of enteric fever. The ceftriaxone, ofloxacin, and gentamycin are found to be $100 \%$ sensitive to Salmonella typhi. Nalidixic acid resistant strains Salmonella Typhi are on the increasing trend. Ampicillin resistance was detected in $65 \%$ of cases. Early diagnosis and instituting appropriate antibiotic therapy has reduced the complication of disease. Pneumonia was found to be the most common complication among all other complications seen in enteric cases.

This study was undertaken to acquire baseline information regarding typhoid fever in Dhulikhel Hospital. Addressing this issue would require a host of measures, including adequate investment in safe water and sanitation services, community education, control over antimicrobial prescribing, and vaccination strategies among others.

\section{Acknowledgement: None \\ Funding: Nil \\ Conflict of Interest: Nil \\ Permission from IRB: Yes}

\section{References}

1. WHO Weekly Epidermiological record 2008;83:4960.

2. Crump JA, Luby S, Mintz E. The global burden of typhoid disease. Bull World Health Org 2004;82:346353.

3. Sharma N, Koju R, Karmacharya B et al. Typhoid fever in Dhulikhel Hospital, Nepal. Kathmandu Univ Med J 2003;2:188-92. 
4. Khanal B, Sharma SK, Bhattacharya SK et al. Antimicrobial susceptibility patterns of Salmonella enteric serotyphi in eastern Nepal. J Health Popul Nutr 2007;25: 82-7.

5. Shakya KN, Baral MR, Shrestha R. A study of atypical manisfestation of enteric fever in children. J Nepal Health Res Council 2008;6: 1-4.

6. WHO Background documents: The diagnosis, treatment and prevention of typhoid fever 2003.4.

7. Rowe B, Threlfall EJ, Ward LR. Does chloramphenicol remain the drug of choice for typhoid? Epidermiol Infect 1987;98:379-83.

8. Bhutta ZA. Typhoid fever. In: Rakel RE, Bope ET, eds. Conn's current therapy. Philadelphia PA: Saunders, 2006:215-8

9. Mohler WT, Levine MD. Salmonella typhi infection in children younger than five years of age. Pediatr Infect Dis J 1993;12:627-31.

10. Rafiq H, Zia R, NaeemSamina Typhoid Fevercontinues as a major threat in children. Biomedica 2009;25:1-2.

11. Malla T, Malla KK, Thapalia L A, Shaw C. Enteric fever: A retrospective 6 year analysis of 82 pediatric cases in a teaching hospital. Kathmandu Univ Med J 2007;5:181-87.

12. Dheer G, KundraS, SinghT. Clinical and laboratory profile of Enteric fever in children in Northern India. Trop Doct 2012Jul;42(3):154-46.

13. Aggrawal A, Ghosh A, Gomber S, Miltra M, Parikh AO. Efficacy and safety of Azithromycin for uncomplicated typhoid fever. Res Brief 2011;28:37772.

14. Ramaswany G, Lalitha J, Thiruvengadam V; Malathi $\mathrm{S}$; Profile of typhoid fever in children from a Tertiary care hospital in Chennai south Indian. J Pediatr 2010;77:1089-1092.

15. Pokharel P, Rai SK, Karki G, Katuwal A, Vitrakoti $R$ and Shrestha SK. Study of enteric fever and antibiogram of Salmonella isolated at a Teaching Hospital in Kathmandu Valley. Nepal Med Coll J 2009;11(3):176178.

16. Amatya NM, Shrestha B, Lekhati B. Etiological agents of bacteraemia and antibiotic susceptibility pattern in Kathmandu Model Hospital. J Nepal Med Assoc 2007;46:112-8.

17. Marks F, Adu-Sarkodie Y, Hunger F et al. Typhoid fever among children, Ghana. Emerg Infect Dis 2010;16(11):176-78.

18. ChauTT, Campbell Jl, Galindo CM, Van Minh Hoang N, Diep TS, Nga TT, et al. Antimicrobial drug resistance of Salmonella enteric serovar Typhi in Asia and molecular mechanism of reduced susceptibility to the fluoroquinolones. Antimicrob Agent Chemother 2007;51:4321-23.

19. Chuang $\mathrm{CH}$, Su LH, Perera J, Carlos C, Tan BH, Kumarasinghe $\mathrm{G}$, et al. Surveillance of antimicrobial resistance of Salmonella enteric serotype typhi in seven Asian countries. Epidemiol Infect 2009;137:266-69.

20. Ochiai LR, Acosta CJ, Danovara Holliday Mc. Baiqing D. Bhattacharya SK et al. A study of typhoid fever in five Asian countries: disease burden and implications for controls. Bull World Health Org 2008;86:241-320.

21. Bhatta CP, Bhuyan KC, Maharjan A. Antibiotic sensitivity pattern of salmonella species isolated from blood culture. J Nepal Health Res Council 2005;3:35-8.

22. Maskalyk J. Typhoid fever. Canadian Med Assoc J 2003;169:2-3.

23. Karkey A, Aryal B, Baker S. Kathmandu, Nepal: still an enteric fever capital of the world. J Infect Dev Ctries 2008;2(6):461-65.

24. Beig FK, Ahmad F, Ekram M, Shukla I. Typhidot M and Diazo test vis-à-vis blood culture and Widal test in the early diagnosis of typhoid fever in children in a resource poor setting. Braz J Infect Dis 2010;14(6):589-93.

\section{How to cite this article?}

Singh DS, Shrestha S, Shrestha N, Manandhar S. Enteric Fever in Children at Dhulikhel Hospital. J Nepal Paediatr Soc 2012;32(3):216-220. 